\title{
Oral nitrite restores age-dependent phenotypes in eNOS-null mice
}

\author{
Margarita Tenopoulou, ${ }^{1}$ Paschalis-Thomas Doulias, ${ }^{1}$ Kent Nakamoto, ${ }^{1}$ Kiara Berrios, ${ }^{1}$ \\ Gabriella Zura, ${ }^{1}$ Chenxi Li, ${ }^{1}$ Michael Faust, ${ }^{1}$ Veronika Yakovishina, ${ }^{1}$ Perry Evans, ${ }^{1}$ Lu Tan, \\ Michael J. Bennett, ${ }^{1}$ Nathaniel W. Snyder, ${ }^{2}$ William J. Quinn III, ${ }^{3}$ Joseph A. Baur, ${ }^{3}$ \\ Dmitriy N. Atochin, ${ }^{4}$ Paul L. Huang, ${ }^{4}$ and Harry Ischiropoulos ${ }^{1}$ \\ 'Children's Hospital of Philadelphia Research Institute, Philadelphia, Pennsylvania, USA. ${ }^{2}$ A.J. Drexel Autism Institute, \\ Drexel University, Philadelphia, Pennsylvania, USA. ${ }^{3}$ Department of Physiology, University of Pennsylvania, Philadelphia, \\ Pennsylvania, USA. ${ }^{4}$ Cardiovascular Research Center Massachusetts General Hospital, Boston, Massachusetts, USA.
}

\begin{abstract}
Alterations in the synthesis and bioavailability of NO are central to the pathogenesis of cardiovascular and metabolic disorders. Although endothelial NO synthase-derived (eNOS-derived) NO affects mitochondrial long-chain fatty acid $\beta$-oxidation, the pathophysiological significance of this regulation remains unclear. Accordingly, we determined the contributions of eNOS/NO signaling in the adaptive metabolic responses to fasting and in age-induced metabolic dysfunction. Four-month-old eNOS-/- mice are glucose intolerant and exhibit serum dyslipidemia and decreased capacity to oxidize fatty acids. However, during fasting, eNOS ${ }^{-/-}$mice redirect acetyl-CoA to ketogenesis to elevate circulating levels of $\beta$-hydroxybutyrate similar to wild-type mice. Treatment of 4-month-old eNOS ${ }^{-1-}$ mice with nitrite for 10 days corrected the hypertension and serum hyperlipidemia and normalized the rate of fatty acid oxidation. Fourteen-month-old eNOS ${ }^{-1-}$ mice exhibited metabolic derangements, resulting in reduced utilization of fat to generate energy, lower resting metabolic activity, and diminished physical activity. Seven-month administration of nitrite to $\mathrm{eNOS}^{-/-}$mice reversed the age-dependent metabolic derangements and restored physical activity. While the eNOS/NO signaling is not essential for the metabolic adaptation to fasting, it is critical for regulating systemic metabolic homeostasis in aging. The development of age-dependent metabolic disorder is prevented by low-dose replenishment of bioactive NO.
\end{abstract}

Authorship note: MT and PTD contributed equally to this work.

Conflict of interest: The authors have declared that no conflict of interest exists.

Submitted: May 10, 2018 Accepted: July 11, 2018 Published: August 23, 2018

Reference information: JCI Insight. 2018;3(16):e122156. https://doi.org/10.1172/jci. insight.122156.

\section{Introduction}

The functions of endothelial cell-derived NO continue to expand following the discovery of NO as a signaling molecule in the cardiovascular system. Emerging evidence indicates that defects in the endothelial NO synthase-derived (eNOS-derived) synthesis and bioavailability of NO are central to the pathogenesis of cardiovascular and metabolic disorders (1-5). Equally, genetic predisposition to enhanced NO signaling offers protection against cardiovascular diseases (6). In addition to vasorelaxation, chronic deficiency in NO signaling influences endothelial function and glucose-insulin and metabolic homeostasis, phenotypes that are recapitulated in the eNOS-null ( $\mathrm{eNOS}^{-/}$) mice. NO deficiency in the $\mathrm{eNOS}^{-/-}$mice leads to systemic hypertension, hyperlipidemia, hepatic steatosis, reduced skeletal muscle capacity to oxidize long-chain fatty acids, and insulin resistance at the level of liver and peripheral tissues under normal diet (7-14). Previously, we reported that eNOS/NO signaling via protein S-nitrosylation has the capacity to regulate the catabolism of long-chain fatty acids in the liver (15). However, the effect of this regulation in normal physiological responses remains unclear. Therefore, we explored for the first time to our knowledge the responses of the $\mathrm{eNOS}^{-/-}$mice to fasting, a well-established model to study metabolic adaptive responses in the liver. During periods of fasting, hepatocytes accommodate a large influx of nonesterified fatty acids that are metabolized inside the mitochondria by $\beta$-oxidation. This catabolic process generates acetyl-CoA that can enter the TCA cycle, sparing glucose and providing energy for gluconeogenesis, or through additional biochemical steps (ketogenesis), can generate metabolic fuel in the form of secreted ketone bodies (acetoacetate, $\beta$-hydroxybutyrate $[\beta-\mathrm{HB}]$, and acetone).

Additional consequences of chronic NO deficiency in aged $\mathrm{NOS}^{-/-}$mice (10 months of age) include alterations in energy metabolism in oxidative skeletal muscle, reduced physical activity, and progressive cardiac dysfunction that may lead to premature death (16-18). These findings and observations in human 
Table 1. Biochemical, metabolic, and hemodynamic parameters in ad libitum-fed 4-month-old wild-type and eNOS ${ }^{-/-}$mice

\begin{tabular}{lcc}
\hline & Wild type & eNOS \\
Body weight $(\mathrm{g})$ & $28.1 \pm 2.7(n=23)$ & $27.0 \pm 3.1(n=21)$ \\
Fat mass $(\mathrm{g})$ & $2.8 \pm 0.9(n=8)$ & $5.9 \pm 3.1^{\mathrm{A}}(n=8)$ \\
Lean mass $(\mathrm{g})$ & $27.2 \pm 2.3(n=8)$ & $24.8 \pm 1.8^{\mathrm{A}}(n=8)$ \\
NO metabolites plasma ( $\mu \mathrm{mol} / \mathrm{l})$ & $33 \pm 4(n=11)$ & $19 \pm 4^{\mathrm{B}}(n=11)$ \\
Diastolic/systolic blood pressure $(\mathrm{mmHg})$ & $120 \pm 25 / 86 \pm 25(n=8)$ & $149 \pm 16^{\mathrm{A}} / 112 \pm 18^{\mathrm{A}}(n=8)$ \\
Mean arterial pressure $(\mathrm{mmHg})$ & $97 \pm 24(n=8)$ & $124 \pm 18^{\mathrm{A}}(n=8)$ \\
Heart rate (beats/min) & $370 \pm 63(n=8)$ & $260 \pm 12^{\mathrm{C}}(n=8)$ \\
Respiratory exchange ratio & $0.98 \pm 0.03(n=8)$ & $0.94 \pm 0.03(n=7)$ \\
Resting metabolic rate (kcal/h/lean mass) - dark & $13.9 \pm 3.1(n=8)$ & $14.5 \pm 2.6(n=8)$ \\
Resting metabolic rate (kcal/h/lean mass) - light & $10.7 \pm 1.5(n=7)$ & $12.8 \pm 2.1(n=7)$ \\
Data were analyzed by 1-way ANOVA, ${ }^{\mathrm{A}} P<0.05,{ }^{\mathrm{B}} P<0.01,{ }^{\mathrm{C}} P<0.001$. & &
\end{tabular}

populations have prompted the development and testing of NO replacement therapies that include the use of inorganic salts of nitrate and nitrite, byproducts of NO metabolism and natural dietary elements $(19,20)$. Restoration of NO signaling with $1 \mathrm{mM}$ nitrate in drinking water for up to 10 weeks reversed the hypertension, systemic hyperlipidemia, and glucose intolerance in 14- to 22 -month-old $\mathrm{eNOS}^{-/-}$mice (21). The use of nitrite in humans is grounded on the known ability of nitrite to dilate isolated blood vessels and original studies testing the efficacy of nitrite to induce vasodilation in humans (22-24). We now appreciate that the acidity of the gastric lumen as well as metalloenzymes produce an array of secondary reactive NO metabolites that elicit production of cGMP as well as formation of protein S-nitrosocysteine after oral administration of nitrite (25-27). Currently, several ongoing clinical trials are testing the efficacy of nitrite as a replenishing NO-based therapy in both acute and chronic cardiovascular disorders. Two clinical trials, NCT02393742 (nitrite supplementation for improving physiological function in older adults) and NCT02918552 (oral nitrite for older heart failure with preserved ejection fraction), target older individuals and aim to improve physical function and capacity, skeletal muscle bioenergetics, and quality of life. Despite these clinical activities, the effects and efficacy of long-term nitrite administration remain unknown. Therefore, using $\mathrm{eNOS}^{-/-}$mice as a relevant preclinical model, we documented that a 7 -month, low-dose of nitrite $(4.6 \mathrm{mg} / 1)$ in the drinking water reversed the age-dependent metabolic deficiencies and physical activity. Collectively, the data document that eNOS/NO signaling is not essential for the metabolic responses to fasting but contributes significantly to age-related phenotypic and metabolic disturbances. Lengthy administration of low-dose nitrite restored metabolic homeostasis and reversed age-dependent phenotypes, providing preclinical evidence for efficacy that we hope is reproduced in the ongoing human trials.

\section{Results}

Phenotypic and metabolic profiling of young eNOS ${ }^{-/}$mice. Ad libitum-fed 4-month-old eNOS ${ }^{-/-}$mice have similar body weight as wild-type mice. Despite similar body weight, the $\mathrm{eNOS}^{-/-}$mice have higher fat and lower lean mass than wild-type mice (Table 1). As it was expected, the deletion of eNOS gene resulted in lower tissue (Supplemental Figure 1A; Supplemental material available online with this article; https://doi. org/10.1172/jci.insight.122156DS1) and circulating levels of NO metabolites; elevated systolic, diastolic, and mean arterial pressures; and bradycardia (Table 1). Indirect calorimetry measurements indicated that $\mathrm{eNOS}^{-/-}$mice have similar oxygen consumption; respiratory exchange ratio (RER), an indicator of metabolic substrate utilization; and resting metabolic rate (Table 1). Despite the similar utilization of metabolic substrates, the $\mathrm{eNOS}^{-/-}$mice had higher levels of circulating triglycerides and nonesterified fatty acids (Figure 1, A and B). In part, the higher fat mass and systemic hyperlipidemia may relate to the lower rate in the catabolism of long-chain fatty acids through $\beta$-oxidation in the liver, skeletal muscle, and heart (Figure 1C). Although these and previous observations $(12,15)$ reinforce the importance of eNOS-derived NO in the regulation of fatty acid metabolism, it remains unclear when this regulation is essential and whether it plays a role in adaptive responses, such as those taking place in the liver during fasting. Therefore, we examined and compared the fasting responses of $\mathrm{eNOS}^{-/-}$to those in wild-type mice. 
A

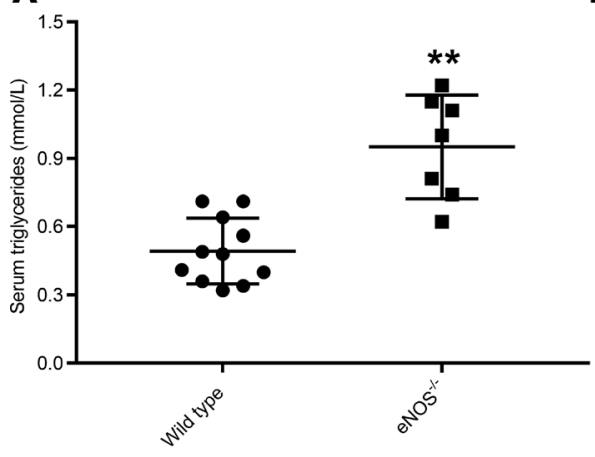

B

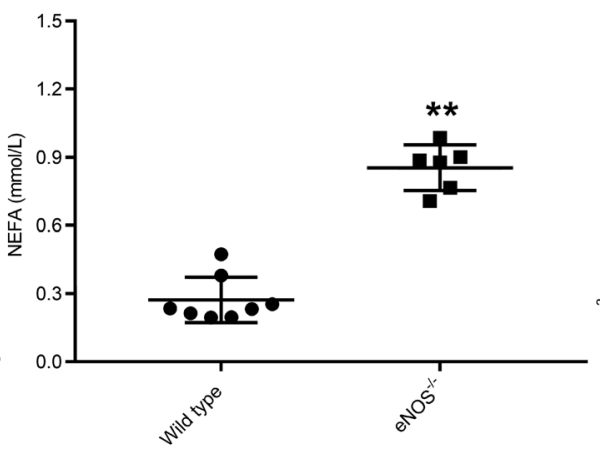

C

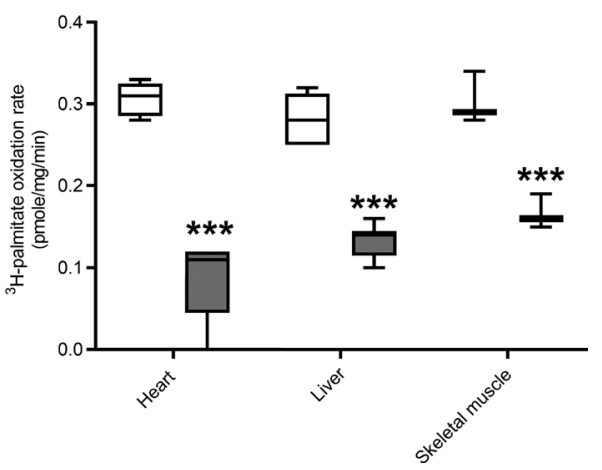

Figure 1. Systemic hyperlipidemia and diminished long-chain fatty acid oxidation capacity in ad libitum-fed 4-month-old eNOS ${ }^{-/-}$mice. (A) Circulating levels of triglycerides and (B) nonesterified fatty acids. (C) Ex vivo quantification of palmitate oxidation rate in the heart, liver, and soleus muscle in wild-type (white boxes) and eNOS ${ }^{-1-}$ (gray boxes) mice $(n=4)$. Scatter dot plots indicate mean \pm SD. Box-and-whisker plots show median, 25 th and 75 th percentiles, and minimum and maximum values. Data were analyzed by 1-way ANOVA, ${ }^{* *} P<0.01,{ }^{* *} P<0.001$ as compared with wild-type mice.

Fasting-induced responses in eNOS ${ }^{-1}$ mice. During periods of prolonged fasting, hepatocytes accommodate a large influx of nonesterified fatty acids by increasing mitochondrial long-chain fatty acid $\beta$-oxidation as well as esterification into triglycerides. Indeed the ex vivo rate of palmitate oxidation in the livers of wild-type mice increased over 3 -fold after fasting, with no significant changes in the $\beta$-oxidation rate in the heart or skeletal muscle (Figure 2A and Supplemental Figure 1B). The rate of palmitate oxidation in the liver did not increase in response to fasting in the $\mathrm{eNOS}^{-/-}$mice. The inability to increase the rate of liver mitochondrial fatty acid oxidation in response to fasting was also documented in mice expressing a mutant eNOS lacking a regulatory site of phosphorylation (eNOS S1176A) but not in mice expressing phosphomimetic S1176D mutant eNOS (Figure 2A). Consistent with the original studies (28), the eNOS S1176A mutant mice had lower levels of NO metabolites as compared with wild-type and the S1176D mutant eNOS mice (Supplemental Figure 1C). Although the data are consistent with the model that eNOS-mediated NO production is required for the increase in longchain fatty acid oxidation rate in response to fasting, the $\mathrm{eNOS}^{-/-}$mice did not display any obvious phenotype after fasting. Consequently, a comprehensive phenotypic, metabolic, and biochemical evaluation of wild-type and $\mathrm{eNOS}^{-/}$mice was performed after 20 hours of fasting followed by a 24 hours refeeding period.

Fasting induced an anticipated decline in body weight and loss of lean and fat mass in both genotypes, although $\mathrm{eNOS}^{-/-}$mice lost less body weight and lean mass during the fasting period (Table 2) and regained less body weight and fat mass in the refed period as compared with age-matched wild-type mice (Supplemental Figure 2A). Both genotypes were able to maintain systemic energy homeostasis, as indicated by the similar RER values under fasting and refed conditions (Figure $2 \mathrm{~B}$ ). Indirect calorimetry measurements indicated that fasted $\mathrm{eNOS}^{--}$mice expend more energy during the light cycle, whereas no differences were documented among genotypes in the dark cycle, irrespective of metabolic state (Supplemental Figure 2, B and C). We also recorded the total and ambulatory activity of mice during the 24-hour dark/light cycle and in response to metabolic state. Both genotypes displayed the same activities (total and forward movements) during the dark and light cycles under fast and refed conditions (Supplemental Figure 2, D-G).

Fasted wild-type and $\mathrm{eNOS}^{-/-}$mice had similar levels of circulating insulin, glucose, and nonesterified fatty acids, whereas triglyceride levels were elevated in $\mathrm{eNOS}^{-/-}$mice as compared with wild-type mice (Table 2). Fasting increased the circulating levels of nonesterified fatty acids in wild-type mice from $0.3 \pm$ $0.1 \mathrm{mM}$ to $0.7 \pm 0.2 \mathrm{mM}$. There was no apparent increase in the circulating levels of nonesterified fatty acids in $\mathrm{eNOS}^{-/-}$mice, which already had elevated levels in the sated state, and these mice showed a lower decline in fat mass upon fasting (Figure 1 and Table 2). The wild-type and $\mathrm{eNOS}^{-/-}$mice had equivalent levels of stored liver glycogen, which upon fasting was depleted to the same extend in both groups (Figure 2C). Contrary to expectations, the systemic levels of $\beta-\mathrm{HB}$, the principal ketone in circulation during fasting, was similar in both groups, and both genotypes exhibited the same drop of $\beta$-HB concentration at the completion of the refed period (Figure 2D). Fasting-induced ketogenesis requires the metabolism of acetyl-CoA derived principally from the $\beta$-oxidation of fatty acids to acetoacetate and $\beta$ - $\mathrm{HB}$. One possible explanation for the ability of eNOS ${ }^{-/}$mice to elevate circulating $\beta$-HB levels during fasting is the redirection of ace- 
A

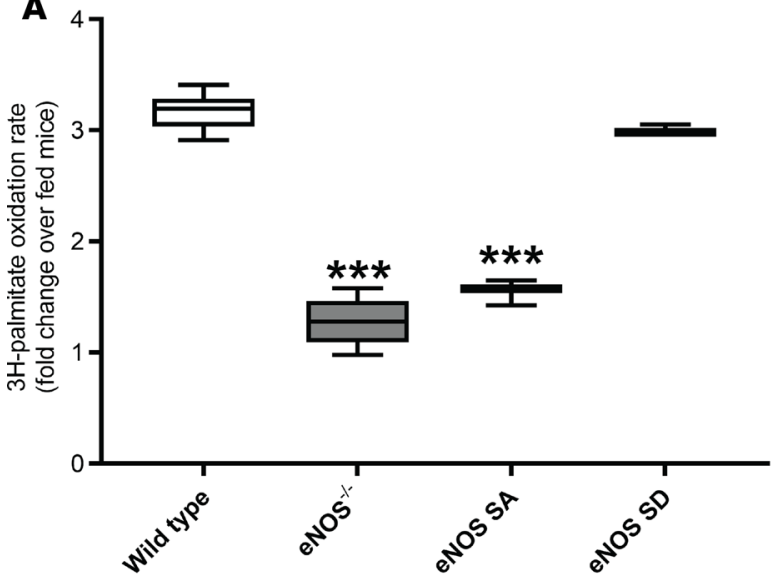

C

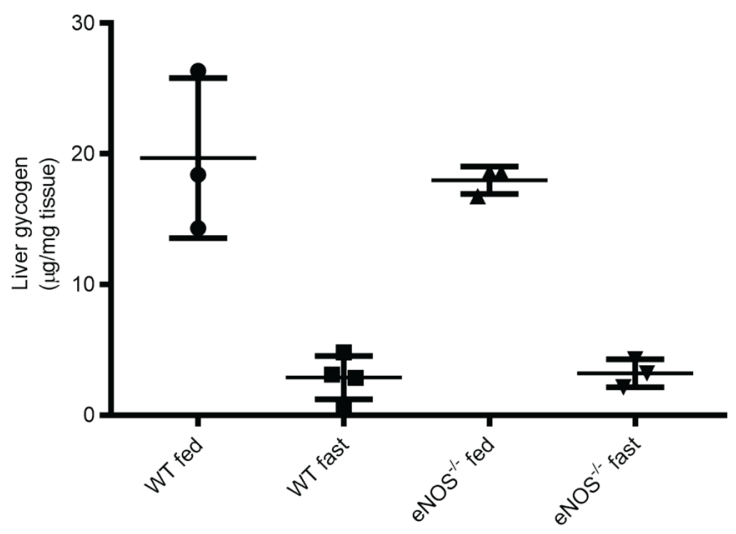

E

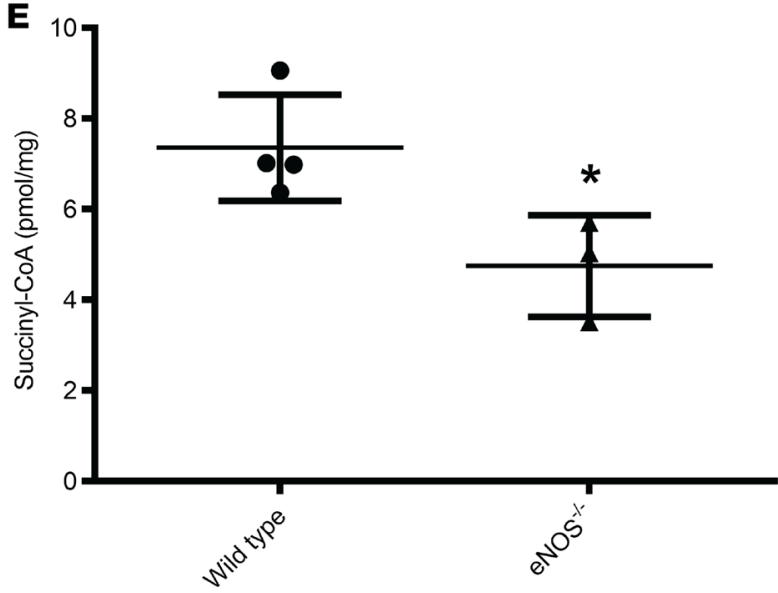

B

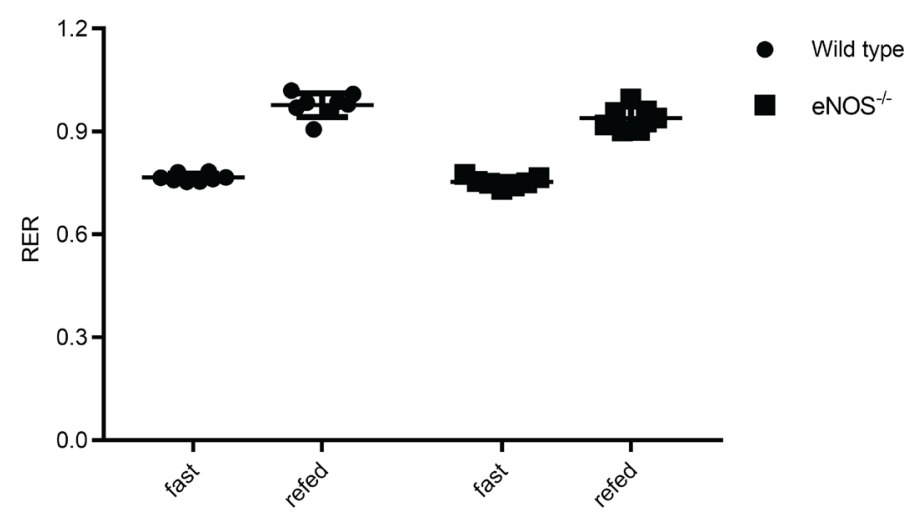

D

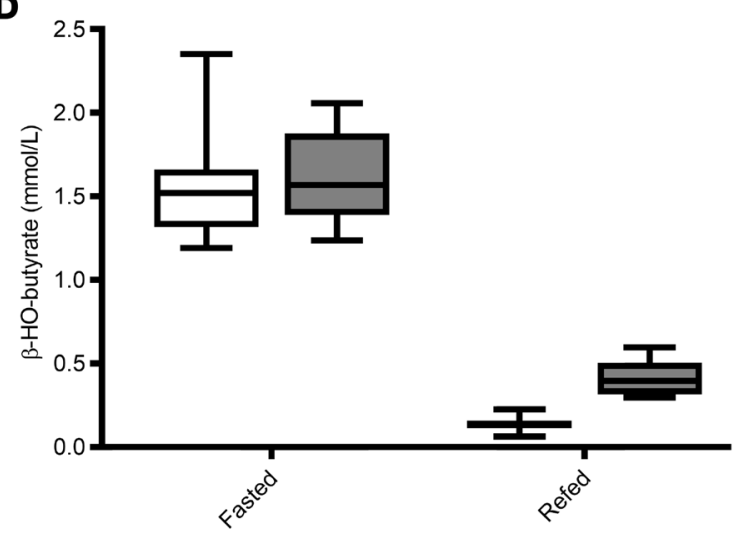

$\mathbf{F}$

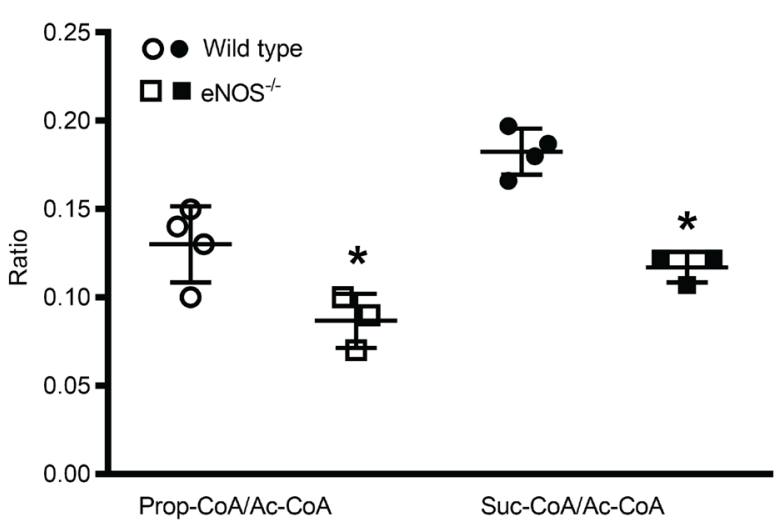

Figure 2. Biochemical and metabolic adaptive responses to fasting are intact in 4-month-old eNOS ${ }^{-1-}$ mice. (A) Ex vivo quantification of palmitate oxidation rate in the livers of wild-type, eNOS ${ }^{-1-}$, eNOS S1176A (eNOS SA), and eNOS S1176D (eNOS SD) mice $(n=4-8)$ after 20 hours of food withdrawal expressed as fold change from the rate quantified at fed state. (B) Respiratory exchange ratio (RER). Circles, wild-type mice; squares, eNOS ${ }^{-1-}$ mice $(n=8)$. (C) Depletion and restoration of liver glycogen levels in response to fasting. (D) Circulating levels of $\beta$-HB. The white boxes correspond to wild-type mice and the gray boxes correspond to eNOS ${ }^{-/-}$mice $(n=8)$. (E) Hepatic levels of succinyl-CoA and $(\mathbf{F})$ the ratios of succinyl-CoA to acetyl-CoA and propionyl-CoA to acetyl-CoA. Scatter dot plots indicate mean $\pm \mathrm{SD}$. Box-and-whisker plots show median, 25 th and 75 th percentiles, and minimum and maximum values. Data were analyzed by 2 -way ANOVA (A-D), and by 1-way ANOVA (E and $\mathbf{F}){ }^{*} P<0.05,{ }^{* * *} P<0.001$.

tyl-CoA to ketogenesis. Using quantitative mass spectrometry-based methodologies, we quantified acylCoA species across several metabolic processes (Supplemental Table 1). These analyses revealed that fasted $\mathrm{eNOS}^{-/-}$mice display significantly lower levels of succinyl-CoA as well as a lower ratio of succinyl-CoA to acetyl-CoA (Figure 2, E and F). The eNOS ${ }^{-/-}$mice also have a reduced ratio of the propionyl-CoA to 
Table 2. Metabolic responses to fasting of 4-month-old wild-type and eNOS-/- mice

\begin{tabular}{lcc}
\hline & Wild type & eNOS $^{-/-}$ \\
Change in body weight $(\mathrm{g})$ & $4.4 \pm 0.6(n=8)$ & $3.2 \pm 0.8^{\mathrm{A}}(n=8)$ \\
Change in fat mass $(\mathrm{g})$ & $0.98 \pm 0.18(n=8)$ & $0.91 \pm 0.2(n=8)$ \\
Change in lean mass $(\mathrm{g})$ & $3.3 \pm 0.7(n=8)$ & $2.6 \pm 0.4^{\mathrm{B}}(n=8)$ \\
Insulin $(\mathrm{ng} / \mathrm{ml})$ & $0.5 \pm 0.1(n=8)$ & $0.4 \pm 0.1(n=8)$ \\
Clucose $(\mathrm{mg} / \mathrm{dl})$ & $84 \pm 10(n=8)$ & $87 \pm 14(n=8)$ \\
Triglycerides (mM) & $0.9 \pm 0.2(n=6)$ & $1.3 \pm 0.3^{\mathrm{B}}(n=6)$ \\
Nonesterified fatty acids $(\mathrm{mM})$ & $0.7 \pm 0.16(n=6)$ & $0.7 \pm 0.2(n=6)$
\end{tabular}

Data were analyzed by 1 -way ANOVA, ${ }^{A} P<0.01,{ }^{\mathrm{B}} P<0.05$.

acetyl-CoA (Figure 2F). Propionyl-CoA can be enzymatically converted to succinyl-CoA and thus serve as an anaplerotic substrate for the TCA cycle. Fasting did not induce the depletion of ketogenic amino acids in the livers of $\mathrm{eNOS}^{-/-}$mice (Supplemental Table 2). Although the $\mathrm{eNOS}^{-/-}$mice were able to adapt metabolically, fasting induced an elevation in liver triglyceride levels (Figure 3A) and in circulating levels of primarily long-chain acylcarnitines, including products of incomplete oxidation of fatty acids, consistent with the reduced $\beta$-oxidation capacity (Figure 3, B-D, and Supplemental Table 3).

These data show that mice deficient in generating NO can adapt to maintain systemic metabolic homeostasis when challenged with fasting but not without possible adverse effects to the liver, resulting from increased content of triglycerides and release of acylcarnitines into circulation.

Phenotypic, metabolic, and biochemical effects of short-term treatment with of sodium nitrite. We administered low levels of sodium nitrite $(100 \mu \mathrm{M}, 4.6 \mathrm{mg} / \mathrm{l})$ into the drinking water for 10-days to 4-month-old wild-type and eNOS $^{-/-}$mice. Nitrite treatment restored the levels of total NO metabolite levels, nitrite and S-nitrosothiols, in the serum, heart, liver, and skeletal muscle of $\mathrm{eNOS}^{-/-}$mice (Figure 4, A and B, and Supplemental Figure 3). Nitrite treatment also reestablished the rate of fatty acid oxidation (Figure 4C) and induced S-nitrosation of very-long-chain acyl CoA dehydrogenase in the heart (Supplemental Figure 4; see complete unedited blots in the supplemental material.). Restoring the rate of long-chain fatty acid oxidation abolished the fasting-induced increase in the levels of circulating acylcarnitine species (Figure 4, D-F, and Supplemental Table 3).

Blood pressure measurements revealed equal values of systolic, diastolic, and mean arterial pressures in wild-type and $\mathrm{eNOS}^{-/-}$mice (Figure $4, \mathrm{G}$ and $\mathrm{H}$ ), indicating that the administration of nitrite corrected the hypertensive phenotype in $\mathrm{eNOS}^{-/-}$mice. Treatment with nitrite corrected the glucose intolerance in the 4-month-old $\mathrm{eNOS}^{-/-}$mice (Figure 4I). $\mathrm{eNOS}^{-/-}$mice treated with nitrite had similar changes in body weight, oxygen consumption, energy expenditure, and physical activity during fed and fasted states as wildtype mice also treated with nitrite (Supplemental Figure 5).

Long-term phenotypic and metabolic effects of sodium nitrite. Fourteen-month-old $\mathrm{eNOS}^{-/-}$mice displayed reduced body weight, lower fat mass, a trend for lower lean mass as compared with age-matched wild-type and $\mathrm{eNOS}^{-/-}$mice treated with nitrite for 7 months (Supplemental Figure 6). Oxygen consumption was measured in the fasted and refed states, RER values were calculated (Supplemental Figure 6), and the percentage of relative cumulative frequency (PRCF) plots were constructed (Figure 5, A and B). The PRCF method for RER data analysis provides a quantitative approach to assess differences in energy metabolism (29). The PRCF curves for each mouse were constructed separately by sorting the indirect calorimetry data in ascending order, calculating their cumulative frequency, which was then plotted against the RER values. The curves of all the mice in each genotype and treatment group were pooled, producing the curves shown in the Figure $5, \mathrm{~A}$ and $\mathrm{B}$, and statistical comparisons and significance for the 50th percentiles were then calculated by a typical $t$ test. $\mathrm{eNOS}^{-/-}$mice were able to adjust the utilization of energy sources, from a mixture of fat and carbohydrates (RER value of $0.84 \pm 0.02$ during fasting) to approaching the theoretical value for pure carbohydrate oxidation (RER value $0.92 \pm 0.04$ ) in refed state. However, these adaptive responses are different from the typical utilization of carbohydrate and fat displayed by age-matched wild-type and nitrite-treated $\mathrm{eNOS}^{-/-}$mice (Figure 5, A and B). The improved metabolic flexibility, the capacity to adapt to the utilization of fuel resources under different nutritional states, in the nitrite-treated $\mathrm{eNOS}^{-/-}$mice was in part due to the restoration of the capacity to oxidize fatty acids $(0.18 \pm 0.01$ versus $0.38 \pm 0.03 \mathrm{pmol} \mathrm{palmitate} / \mathrm{min} / \mathrm{mg}$ liver protein, for untreated and nitrite-treated mice, respectively). The $\mathrm{eNOS}^{-/-}$mice exhibited lower resting meta- 
A

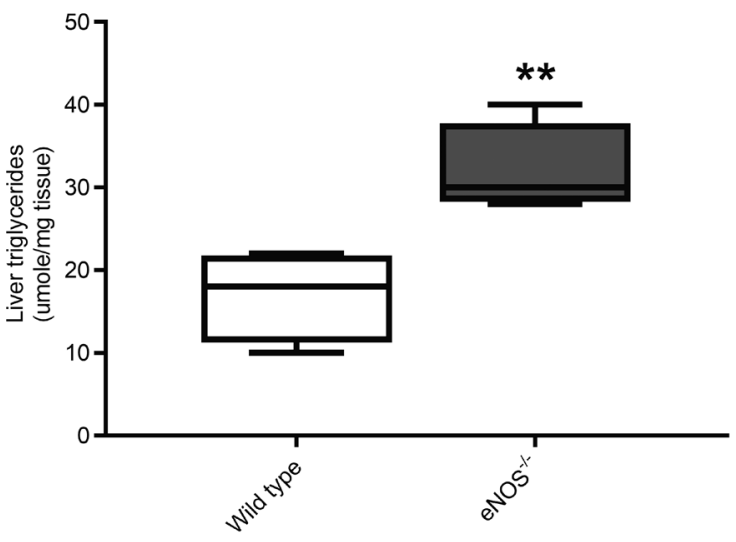

C

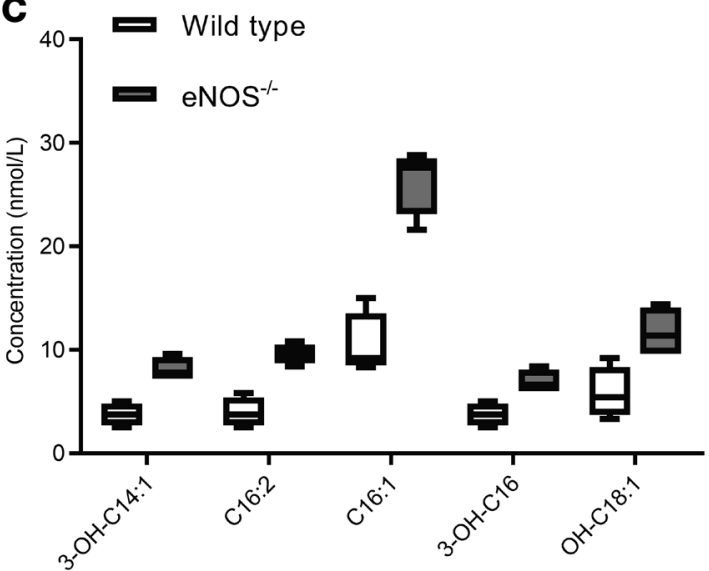

B

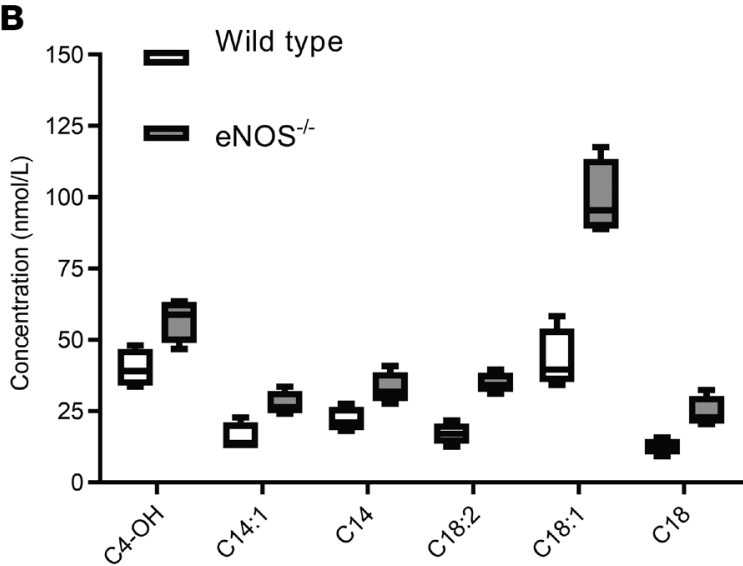

D

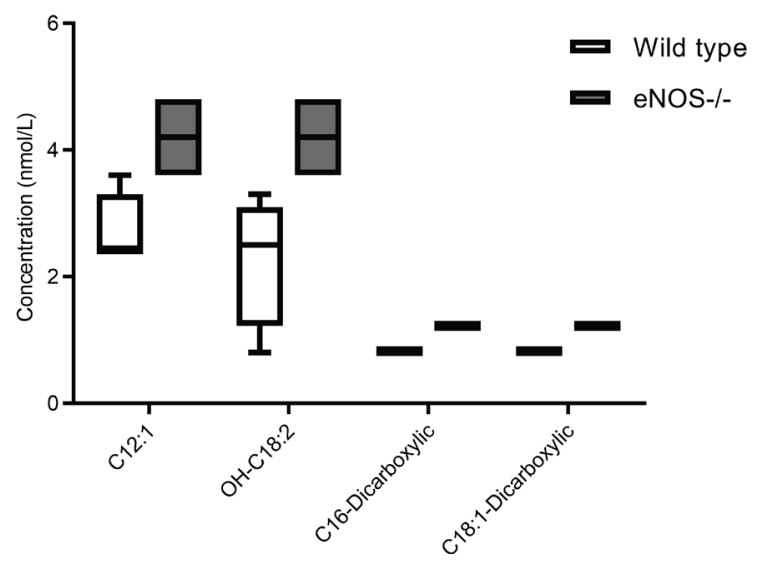

Figure 3. Fasting induced increased liver triglycerides and increased circulating acylcarnitines in 4-month-old eNOS ${ }^{-1-}$ mice. (A) Liver triglyceride levels after 20 hours of food withdrawal. Box-and-whisker plots show median, 25th and 75th percentiles, and minimum and maximum values $(n=4)$. Data were analyzed by 1-way ANOVA, ${ }^{* *} P<0.01$. (B-D) Circulating levels of acylcarnitines that are significantly elevated in the sera of eNOS ${ }^{-/-}$mice (gray boxes) as compared with wild-type (white boxes) mice after 20 hours of fasting. Box-and-whisker plots show median, 25 th and 75 th percentiles, and minimum and maximum values ( $n$ =4). Data were analyzed by 1-way ANOVA, $P<0.05$.

bolic rate during 24-hour monitoring, which coincided with significantly lower ambulatory activity (Figure 5, $\mathrm{C}$ and D). Long-term treatment with nitrite of $\mathrm{eNOS}^{-/-}$mice attenuated the deficits in energy expenditure and critically in ambulatory activity (Figure $5, \mathrm{C}$ and D). Collectively, the data document that aged $\mathrm{eNOS}^{-/-}$mice have significant metabolic derangements and become physically inactive. The biochemical, metabolic, and phenotypic changes in aged $\mathrm{eNOS}^{-/-}$nice are prevented through the administration of nitrite.

\section{Discussion}

Several key observations in humans and animal models indicate essential contributions of the precisely regulated enzymatic production of $\mathrm{NO}$ in cardiovascular and metabolic processes. NO provides spatial and temporal signaling by activating soluble guanylate cyclase and production of cGMP, which, in turn, controls phosphorylation and dephosphorylation signaling events. NO signaling is also accomplished through S-nitrosylation, a selective posttranslational modification of protein cysteine residues (30-32). Although the expression and activity of eNOS in nonvascular endothelial cells has been documented in metabolically active organs, such as the liver, heart, and skeletal muscle, it remains unclear if eNOS-derived NO and downstream signaling coordinates biological responses to physiological stimuli. To explore the significance of eNOS/NO signaling in regulating systemic metabolic homeostasis, we explored the responses of $\mathrm{eNOS}^{-/-}$mice to fasting, a physiologically relevant stimulus. To provide additional evidence that the eNOS/NO signaling is relevant for the homeostatic regulation, we restored bioavailable $\mathrm{NO}$ in the $\mathrm{eNOS}^{-/-}$mice and determined the changes in metabolic utilization of substrates and, critically, the consequences in the development of age-dependent metabolic phenotypes.

We decided to study the fasting response, since we and others have documented the decreased capacity 
A
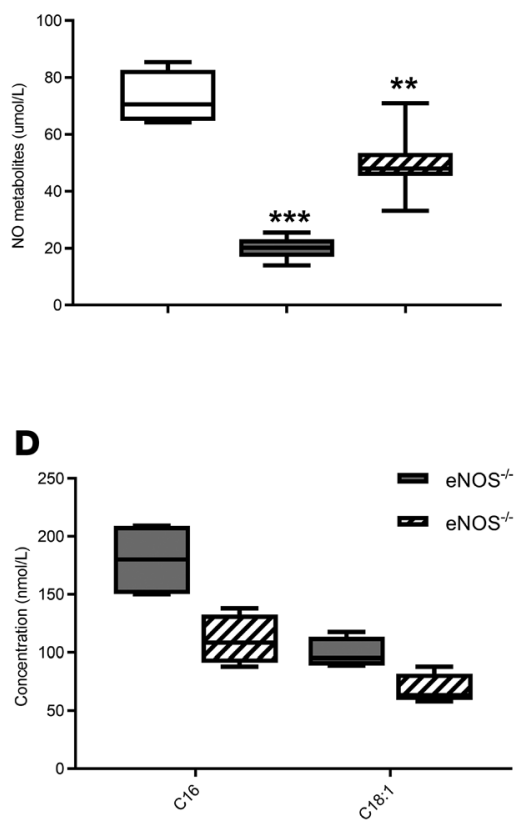

G

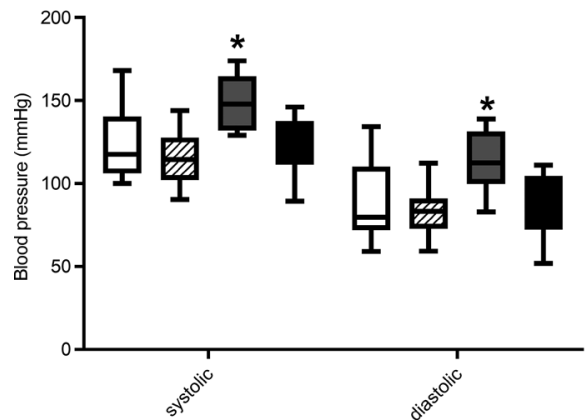

B

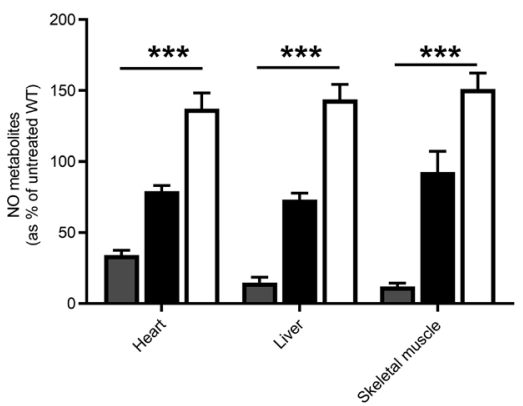

E

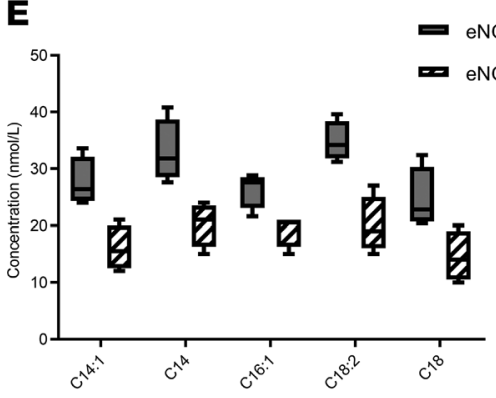

H

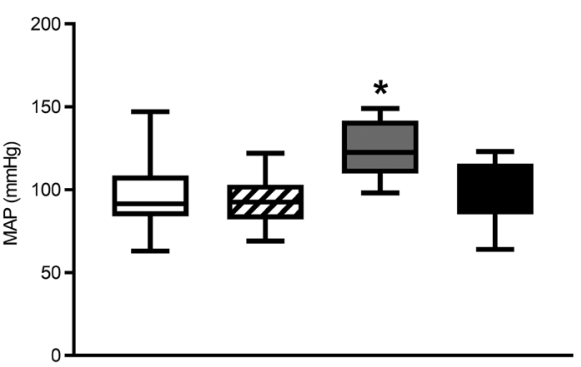

C

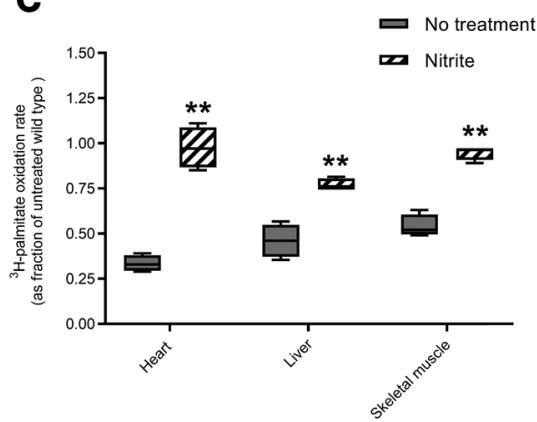

F

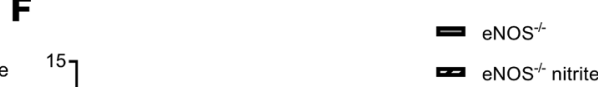

Figure 4. Ten-day administration of $\mathrm{NaNO}_{2}$ restores biochemical and metabolic phenotypes in 4-month-old eNOS ${ }^{-/-}$mice. Treatment with $100 \mu \mathrm{M}$ nitrite in the drinking water restored (A) serum levels of NO metabolites. eNOS ${ }^{-1-}$ mice (gray box) display lower levels of serum NO metabolites as compared with wild-type mice treated with nitrite (white box) $(n=4-6)$. Data were analyzed by 1 -way ANOVA, ${ }^{* * *} P<0.001$. Serum NO metabolites in eNOS 1- mice treated with nitrite (hatched box) were significantly higher than untreated eNOS ${ }^{-1-}$ mice (data were analyzed by 1 -way ANOVA, ${ }^{* * *} P<0.001$ ) and lower than wild-type mice treated with nitrite (data were analyzed by 1-way ANOVA, ${ }^{* *} P<0.01$ ). (B) Tissue NO metabolite levels in eNOS ${ }^{-/-}$mice treated with nitrite (black) were significantly higher than untreated eNOS ${ }^{-1-}$ mice (gray) and lower than wild-type mice (white) treated with nitrite eNOS $\mathrm{S}^{-1-}$ mice (data were analyzed by 1-way ANOVA, $\left.{ }^{* *} P<0.001\right)(n=4-6)$. Tissue NO metabolites are expressed as the percentage of levels in untreated wild-type mice. (C) Ex vivo palmitate oxidation rate expressed as a fraction of the rate in wild-type mice. Nitrite treatment of eNOS ${ }^{-1-}$ mice (hatched box) restored the rate of palmitate oxidation. Gray boxes show the rate of untreated eNOS ${ }^{-1-}$ mice (data were analyzed by 1-way ANOVA, $\left.{ }^{* *} P<0.01\right)(n=4-6)$. (D-F) Exposure to $100 \mu \mathrm{M}$ nitrite in the drinking water normalized the levels of circulating acylcarnitines. Gray boxes, eNOS ${ }^{-1-}$ without nitrite; hatched boxes, eNOS $^{-1-}$ with nitrite $(n=4)$. (G) Blood pressure, $(\mathbf{H})$ mean arterial pressure $(\mathrm{MAP})$, and $(\mathbf{I})$ glucose tolerance in eNOS ${ }^{-1-}$ mice. In $\mathbf{G}$ and $\mathbf{H}$, white and hatched boxes correspond to untreated and nitrite-treated wild-type mice, respectively. Gray and black boxes correspond to untreated and nitrite-treated eNOS ${ }^{-1-}$ mice. In I, eNOS ${ }^{-1-}$ mice displayed significantly greater levels of blood glucose as compared with wild-type, wild-type treated with nitrite, and eNOS ${ }^{-/-}$ treated with nitrite groups at 15, 30, and 60 minutes (data were analyzed by 1-way ANOVA, $P<0.001$ for all groups and time points) ( $n=7-8$ ).

of $\mathrm{eNOS}^{-/-}$mice to oxidize long-chain fatty acids in the skeletal muscle (12), liver (15), and heart (this work). Fatty acid oxidation is an essential metabolic process in responses to nutrient deprivation. During fasting, the liver increases its capacity to oxidize long chain fatty acids to acetyl-CoA, as shown for wild-type mice after 20 hours of food withdrawal. This increase was absent in the $\mathrm{eNOS}^{-/-}$mice and in mutant eNOS mice lacking a regulatory site of phosphorylation (eNOS S1176A) but not in the phosphomimetic S1176D mutant mice. Despite the significantly lower capacity for complete oxidation of fat-derived carbons, 4-month-old eNOS ${ }^{-/}$ mice were able to satisfy their peripheral metabolic needs by increasing the circulating levels of $\beta$-HB. In part, eNOS $^{-/-}$mice may have adapted to this demand by redirecting acetyl-CoA toward ketogenesis as opposed to the TCA cycle. Although eNOS/NO signaling is not essential for maintaining acute systemic homeostatic 
$\mathbf{A}$

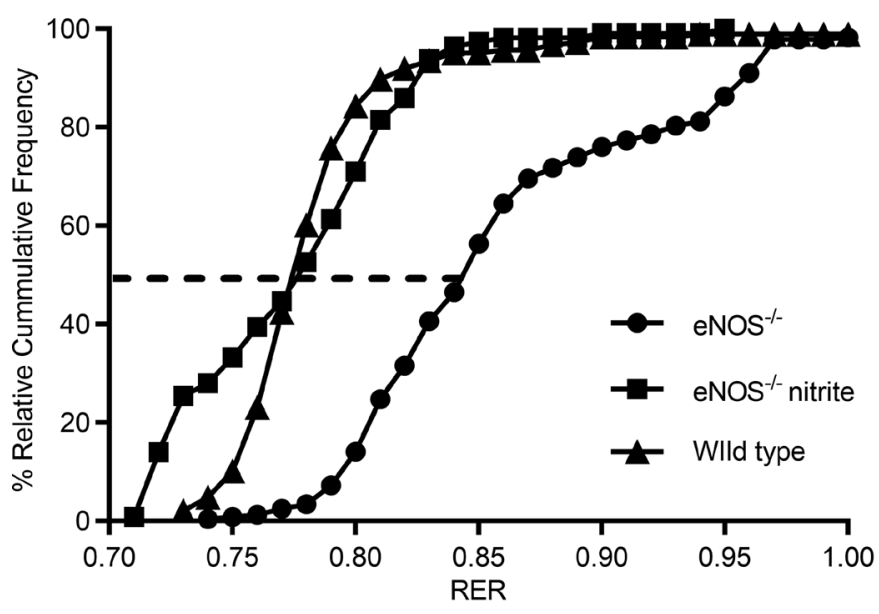

$\mathbf{B}$

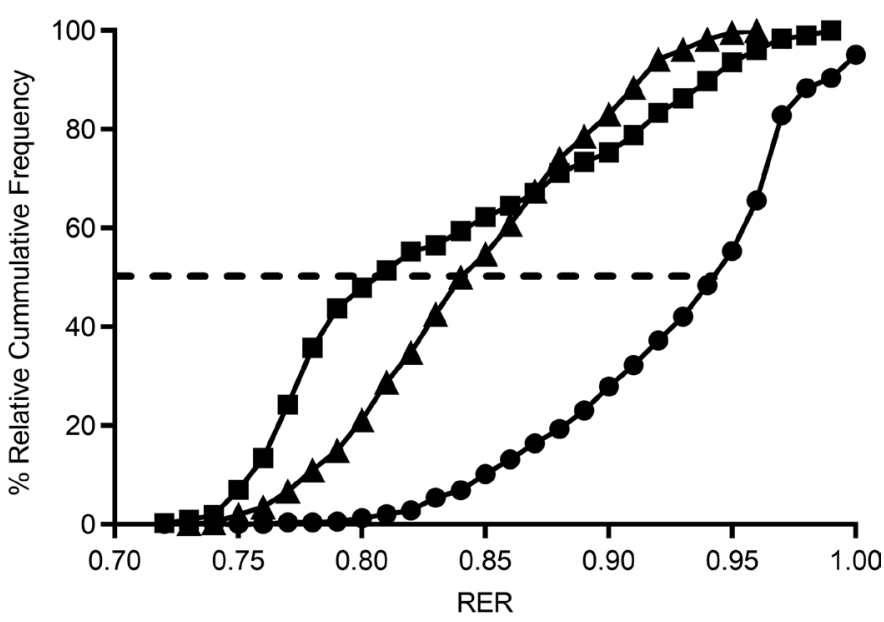

D

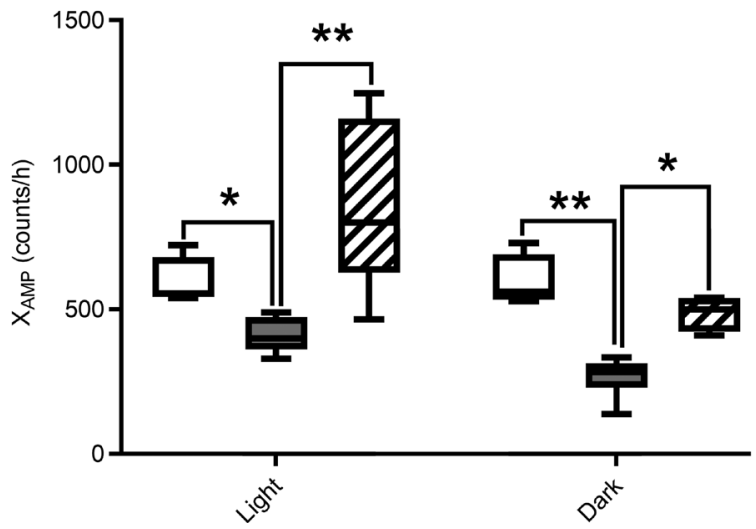

Figure 5. Long-term treatment with sodium nitrite restored metabolic flexibility and age-dependent phenotypes in eNOS ${ }^{-/-}$mice. Seven-month-old $\mathrm{eNOS}^{-1-}$ mice were treated with $100 \mu \mathrm{M}$ nitrite in the drinking water for 7 months. Nonnitrite-treated age-matched wild-type mice were used as controls. (A) Cumulative frequency of respiratory exchange values after 6 hours of food withdrawal. (B) Cumulative frequency of respiratory exchange values after 24 hours of refeeding ( $n=5-7)$. (C) Resting metabolic activity under fed conditions during the light and dark periods. (D) Ambulatory activity during the 24-hour dark and light cycles. White boxes, wild-type mice $(n=4)$; gray boxes, eNOS ${ }^{-1-}$ without nitrite; and hatched boxes, eNOS ${ }^{-1-}$ with nitrite $(n=5-7)$. Box-and-whisker plots show median, 25th and 75th percentiles, and minimum and maximum values. Data were analyzed by 1-way ANOVA, ${ }^{*} P<0.05$, ${ }^{* *} P<0.01$.

responses to a physiological stimulus, the failure to increase oxidative capacity may negatively influence the liver and systemic metabolic homeostasis. The increase in liver triglyceride levels and the release of acylcarnitines into the circulation in the $\mathrm{eNOS}^{-/-}$mice imply that the liver is adapting to the apparent stress induced by the diminished capacity for long-chain fatty acid oxidation. This response may be critical, since a body of evidence supports the idea that incomplete and inefficient mitochondrial fatty acid $\beta$-oxidation leads to the accumulation and diversion of lipid precursors and partially oxidized lipid species into the cytosol and ER, causing stress and leading to the development of hepatic insulin resistance (33-35). Moreover, $\mathrm{eNOS}^{-/-}$mice on a high-fat diet for 3 months develop early signs of nonalcoholic fatty liver disease and a risk factor for type 2 diabetes, metabolic syndrome, and other liver complications (36).

However, over time the insufficient metabolism of long-chain fatty acids in the $\mathrm{eNOS}^{-/-}$mice may contribute to the age-dependent progression of metabolic dysfunction and development of pathological phenotypes. Our data support a critical role for NO-mediated signaling in the regulation of metabolic homeostasis by documenting that chronic, low-level nitrite treatment of $\mathrm{eNOS}^{-/-}$mice prevents age-induced metabolic phenotypes. In addition to hypertension, $\mathrm{eNOS}^{-/-}$mice develop progressive and age-dependent heart defects, hyperlipidemia, hepatic steatosis, and insulin resistance at the level of liver and peripheral tissues under standard chow feeding (7-14). These phenotypic changes are also reproduced by administration 
of NOS inhibitors in the food of wild-type rats, reaffirming that the decline in bioavailable NO and NO signaling is responsible for the metabolic changes $(37,38)$. The data presented in Figure 5 confirmed the age-dependent reduction in energy expenditure and oxygen consumption $(16,17)$ and indicated an increase reliance on carbohydrate utilization in aged $\mathrm{eNOS}^{-/-}$mice, consistent with the inability to utilize fatty acid oxidation for energy production. Under fed conditions, $\mathrm{eNOS}^{-/}$mice generate higher proportions of energy from carbohydrate oxidation, whereas $\mathrm{eNOS}^{-/}$mice treated with nitrite show a pattern of substrate utilization similar to that of age-matched wild-type controls. The metabolic derangement and reduction in energy expenditure are associated with reduced mobility. Treatment with nitrite for 7 months increased the systemic and tissue levels of NO metabolites, restored the capacity to metabolize fatty acids, improved the physical activity in both the light and dark cycles, and restored metabolic homeostasis. Although in our study the $\mathrm{eNOS}^{-/-}$mice showed increased adiposity, their body weight declined as these mice became less active, which is consistent with previous reports indicating that aged male $\mathrm{eNOS}^{-/-}$mice have similar or decreased body weight compared with age-matched controls, develop cardiac insufficiency, and die prematurely (18). Although typical pharmacological applications of nitrite employ higher concentrations (100-100 mg/1), we decided to use a low dose and extend the administration period to 7 months, aiming to investigate the long-term effects and efficacy of replenishing bioactive NO in a relatively physiological context. Overall, the data suggest that the chronic replenishment of bioavailable NO prevented the biochemi$\mathrm{cal}$, metabolic, and phenotypic decline in $\mathrm{eNOS}^{-/-}$mice, indicating the critical influence of the eNOS/NO signaling in maintaining metabolic homeostasis. Although trials with nitrite are ongoing, our preclinical findings in $\mathrm{eNOS}^{-/-}$mice support the notion that long-term nitrite treatment may be a suitable approach to correct the metabolic and phenotypic alterations, such as hypertension and metabolic syndrome.

\section{Methods}

Animal studies. The studies were performed in male C57/BL6J (stock 000664) and NOS3-null (eNOS ${ }^{-/-}$) mice (Nos3 $3^{\text {tm IUnc }}\left[\mathrm{eNOS}^{-/}\right]$C57BL/6J, stock 002684) from The Jackson Laboratory. The eNOS ${ }^{-/-}$mice were backcrossed to the C57/BL6J background for at least 12 generations. Liver tissues from the eNOS S1176A and eNOS S1176D mice were obtained as described previously (20). Based on previous publications that have explored the responses of different mouse strains to fasting and the various phenotypes in eNOS ${ }^{-1}$ mice, we decided to use male mice fed standard chow diet (39). Mice were studied at the ages of 4 months and between 13 and 15 months. For fasting metabolic studies, mice were deprived of food for 6 hours (aged mice) or 20 hours (young mice) from $4 \mathrm{pm}$ to $10 \mathrm{am}$, and, after continuous monitoring, the mice were sacrificed at the same time to ensure consistency. For the nitrite treatment, the regular water was supplemented with $100 \mu \mathrm{M}$ (4.6 mg/1) sodium nitrite. The 4-month-old mice were treated for 10 days. Treatment of the older mice started at 6 or 7 months of age and continued for an additional 7 months.

Metabolic monitoring, MRI for body composition, glucose tolerance test, and blood pressure measurements. Mice were individually placed in the Oxymax Lab Animal Monitoring System (Columbus Instruments) with free access to food and water. After overnight acclimation, oxygen consumption, the amount of carbon dioxide produced, food and drink intake, and locomotor activity (beam breaks) were determined during ad libitum, fasting, and refeeding conditions. Fat and lean mass determinations were performed using the Eco-MRI-100 system (Echo Medical Systems). For the glucose tolerance test, mice were fasted for 16 hours followed by intraperitoneal injection of glucose ( $2 \mathrm{~g} / \mathrm{kg}$ body weight), and blood glucose was monitored at $0,15,30,60$, and 120 minutes after injection using a Rely-On handheld glucose monitoring system (Rely-On). For blood pressure measurements, mice were placed into restrainers and tails were threaded into blood pressure cuffs. Tails were then heated to $35^{\circ} \mathrm{C}$, and blood pressure and heart rate were monitored using the CODA 8 noninvasive blood pressure monitoring system (Kent Scientific). RERs were calculated by dividing the volume of carbon dioxide produced by the volume of oxygen consumed $\left(\mathrm{VCO}_{2} / \mathrm{VO}_{2}\right)$. The PRCF, which allows for the systematic examination and comparison of large data sets collected by indirect calorimetry for each mouse, was calculated as described previously (29). The 8 curves from each mouse were pooled and plotted against RER.

Quantification of triglycerides, free fatty acids, glycogen, acylcarnitines, ketones, acyl-CoA species, and ex vivo fatty acid oxidation assay. Triglycerides, nonesterified free fatty acids, $\beta-\mathrm{HB}$, and glycogen were quantified using established colorimetric assays (15). For quantification, standard curves using authentic triglycerides, free fatty acids, and glycogen were employed. For glycogen quantification, glycogen was hydrolyzed by glucoamylase to glucose, which is then oxidized and reacts with the color probe to generate a product that is measured at $570 \mathrm{~nm}$. Acylcarnitines were measured, as their butylated derivatives using flow injection tandem 
mass spectrometry and stable isotope dilution quantitation as described previously (40). Liver acyl-CoA levels were quantified by liquid chromatography-mass spectrometry using previously validated methods $(40,41)$. Briefly, 50 -mg sections of liver were homogenized by pulse sonication in $10 \%$ trichloroacetic acid containing ${ }^{13} \mathrm{C}_{3}{ }^{15} \mathrm{~N}_{1}$-labeled internal standards generated as previously described. Calibration curves were generated in parallel using standards from MilliporeSigma. Samples were analyzed in a blinded manner on an Ultimate 3000 coupled to a Q Exactive Plus high-resolution mass spectrometer with data analysis and instrument control by XCalibur software (Thermo Fisher Scientific). The rate of fatty acid oxidation was performed in tissue homogenates obtained from tissue extraction in buffer (250 mM HEPES-NaOH, $\mathrm{pH}$ 7.7, containing $1 \mathrm{mM}$ DTPA, $0.1 \mathrm{mM}$ neocuproine, $1 \%$ Triton X-100, and protease inhibitors) on ice using a Teflon pestle and a Jumbo Stirrer (Fisher Scientific). The homogenates were then centrifuged at 13,000 $\mathrm{g}$ for 30 minutes at $4^{\circ} \mathrm{C}$. The soluble protein fraction was collected, and the protein concentration was determined by the Bradford assay. Homogenates were incubated with $[9,10-3 \mathrm{H}]$ palmitate as previously described (15) followed by chloroform/methanol and strong anion exchange chromatographic separation of ${ }^{3} \mathrm{H}$-palmitate and ${ }^{3} \mathrm{H}_{2} \mathrm{O}$ that is generated through the oxidation of palmitate. Scintillation counts are converted to amount of palmitate using a titration curve constructed by authentic ${ }^{3} \mathrm{H}$-palmitate.

NO metabolites. The quantification of NO metabolites concentration in plasma and tissue was performed using reductive chemistries coupled with ozone-based chemiluminescence detection of NO. NO metabolites, namely nitrate, nitrite, low-molecular-weight S-nitrosothiols, lipid-bound NO, and metal-bound NO, were initially reduced by vanadium (III) under acidic conditions $(1 \mathrm{~N} \mathrm{HCL})$ at $95^{\circ} \mathrm{C}$. The $\mathrm{NO}$ that is liberated in the gas phase reacts with ozone-forming nitrogen dioxide in an excited state. When the nitrogen dioxide returns to the ground state, it releases a photon that is quantified by a photomultiplier tube and the emitted light is transformed to electrical signal, which is recorded. Signals are converted to concentration of metabolites using a standard curve constructed by authentic nitrate. Nitrite and S-nitrosothiols were quantified in acidified tri-iodide solution at $37^{\circ} \mathrm{C}$ as previously described (42). To quantify S-nitrosothiols, plasma and tissue homogenates were treated with $1 \%$ sulfanilamide (SNA) for 10 minutes before the injection. To discriminate S-nitrosothiols and other NO-related species, SNA-treated plasma and tissue homogenates were incubated with 5 $\mathrm{mM} \mathrm{HgCl}$ for 10 minutes prior to injection. The $\mathrm{HgCl}_{2}$-sensitive signal was used to quantify S-nitrosothiols.

Very-long-chain acyl-CoA dehydrogenase S-nitrosation. The S-nitrosation of very-long-chain acyl-CoA dehydrogenase was determined as it was described previously (15). Rabbit polyclonal antibodies against very-longchain acyl-CoA dehydrogenase (clone N1C1, $1 \mathrm{mg} / \mathrm{ml}$, protein GTX114232) were obtained from Genetex. The antibody was diluted in $1 \%$ nonfat milk in TBST at a final concentration of $0.25 \mu \mathrm{g} / \mathrm{ml}$ and was incubated with the membranes overnight at $4^{\circ} \mathrm{C}$. All chemicals and reagents used were of analytical grade.

Statistics. Data were analyzed with GraphPad Prism 7.04 software. All normally distributed data were displayed as mean \pm SD. Groups were analyzed by 1-way or 2-way ANOVA. Multiple comparisons were performed using the Tukey multiple comparisons post hoc test and 2-tailed unpaired $t$ tests. A $P$ value less than 0.05 was considered significant.

Study approval. All procedures were performed in accordance with the National Institute of Health Guide for the Care and Use of Laboratory Animals (National Academies Press, 2011), after receiving approval from the Children's Hospital of Philadelphia Research Institute and the University of Pennsylvania Animal Care and Use Committees.

\section{Author contributions}

MT, PTD, WJQ, JAB, PLH, and HI conceived and designed the experiments; MT, PTD, KN, KB, GZ, CL, MF, VY, LT, MJB, NWS, WJQ, and DNA performed the experiments, analyzed data, and provided discussions; PE performed statistical analysis; and $\mathrm{HI}$ wrote the paper, with important contributions from MT, PTD, WJQ, and JAB.

\section{Acknowledgments}

We thank Itzhak Nissim at the Metabolomics Core at the Children's Hospital of Philadelphia Research Institute for the amino acid analysis and Payman Zamani for comments and suggestions. National Institutes of Health grant HL054926 and National Institute of Environmental Health Sciences Center of Excellence in Environmental Toxicology grant ES013508 supported this work. HI is the Gisela and Dennis Alter Research Professor of Pediatrics. 
Address correspondence to: Harry Ischiropoulos, Children's Hospital of Philadelphia Research Institute, 417 Abramson Research Center, 3517 Civic Center Boulevard, Philadelphia, Pennsylvania, 19104-4318, USA. Phone: 215.590.5320; Email: ischirop@pennmedicine.upenn.edu.

1. Huang PL. eNOS, metabolic syndrome and cardiovascular disease. Trends Endocrinol Metab. 2009;20(6):295-302.

2. Johnstone MT, Creager SJ, Scales KM, Cusco JA, Lee BK, Creager MA. Impaired endothelium-dependent vasodilation in patients with insulin-dependent diabetes mellitus. Circulation. 1993;88(6):2510-2516.

3. Petrie JR, Ueda S, Webb DJ, Elliott HL, Connell JM. Endothelial nitric oxide production and insulin sensitivity. A physiological link with implications for pathogenesis of cardiovascular disease. Circulation. 1996;93(7):1331-1333.

4. Siervo M, Jackson SJ, Bluck LJ. In-vivo nitric oxide synthesis is reduced in obese patients with metabolic syndrome: application of a novel stable isotopic method. J Hypertens. 2011;29(8):1515-1527.

5. Erdmann J, et al. Dysfunctional nitric oxide signalling increases risk of myocardial infarction. Nature. 2013;504(7480):432-436.

6. Emdin CA, et al. Phenotypic consequences of a genetic predisposition to enhanced nitric oxide signaling. Circulation. 2018;137(3):222-232.

7. Huang PL, et al. Hypertension in mice lacking the gene for endothelial nitric oxide synthase. Nature. 1995;377(6546):239-242.

8. Shesely EG, et al. Elevated blood pressures in mice lacking endothelial nitric oxide synthase. Proc Natl Acad Sci USA. 1996;93(23):13176-13181.

9. Shankar RR, Wu Y, Shen HQ, Zhu JS, Baron AD. Mice with gene disruption of both endothelial and neuronal nitric oxide synthase exhibit insulin resistance. Diabetes. 2000;49(5):684-687.

10. Duplain $\mathrm{H}$, et al. Insulin resistance, hyperlipidemia, and hypertension in mice lacking endothelial nitric oxide synthase. Circulation. 2001;104(3):342-345.

11. Cook S, et al. Partial gene deletion of endothelial nitric oxide synthase predisposes to exaggerated high-fat diet-induced insulin resistance and arterial hypertension. Diabetes. 2004;53(8):2067-2072.

12. Le Gouill E, et al. Endothelial nitric oxide synthase (eNOS) knockout mice have defective mitochondrial beta-oxidation. Diabetes. 2007;56(11):2690-2696.

13. Mohan S, et al. Diabetic eNOS knockout mice develop distinct macro- and microvascular complications. Lab Invest. 2008;88(5):515-528.

14. Schild L, Dombrowski F, Lendeckel U, Schulz C, Gardemann A, Keilhoff G. Impairment of endothelial nitric oxide synthase causes abnormal fat and glycogen deposition in liver. Biochim Biophys Acta. 2008;1782(3):180-187.

15. Doulias PT, Tenopoulou M, Greene JL, Raju K, Ischiropoulos H. Nitric oxide regulates mitochondrial fatty acid metabolism through reversible protein S-nitrosylation. Sci Signal. 2013;6(256):rs1.

16. Momken I, Fortin D, Serrurier B, Bigard X, Ventura-Clapier R, Veksler V. Endothelial nitric oxide synthase (NOS) deficiency affects energy metabolism pattern in murine oxidative skeletal muscle. Biochem J. 2002;368(Pt 1):341-347.

17. Momken I, Lechêne P, Ventura-Clapier R, Veksler V. Voluntary physical activity alterations in endothelial nitric oxide synthase knockout mice. Am J Physiol Heart Circ Physiol. 2004;287(2):H914-H920.

18. Li W, Mital S, Ojaimi C, Csiszar A, Kaley G, Hintze TH. Premature death and age-related cardiac dysfunction in male eNOS-knockout mice. J Mol Cell Cardiol. 2004;37(3):671-680.

19. Lundberg JO, et al. Nitrate and nitrite in biology, nutrition and therapeutics. Nat Chem Biol. 2009;5(12):865-869.

20. Lundberg JO, Gladwin MT, Weitzberg E. Strategies to increase nitric oxide signalling in cardiovascular disease. Nat Rev Drug Discov. 2015;14(9):623-641.

21. Carlström M, et al. Dietary inorganic nitrate reverses features of metabolic syndrome in endothelial nitric oxide synthase-deficient mice. Proc Natl Acad Sci USA. 2010;107(41):17716-17720.

22. Furchgott RF, Bhadrakom S. Reactions of strips of rabbit aorta to epinephrine, isopropylarterenol, sodium nitrite and other drugs. J Pharmacol Exp Ther. 1953;108(2):129-143.

23. Gladwin MT, et al. Role of circulating nitrite and S-nitrosohemoglobin in the regulation of regional blood flow in humans. Proc Natl Acad Sci USA. 2000;97(21):11482-11487.

24. Cosby K, et al. Nitrite reduction to nitric oxide by deoxyhemoglobin vasodilates the human circulation. Nat Med. 2003;9(12):1498-1505.

25. Zweier JL, Wang P, Samouilov A, Kuppusamy P. Enzyme-independent formation of nitric oxide in biological tissues. Nat Med. 1995;1(8):804-809.

26. Bryan NS, et al. Nitrite is a signaling molecule and regulator of gene expression in mammalian tissues. Nat Chem Biol. 2005;1(5):290-297.

27. Chouchani ET, et al. Identification and quantification of protein S-nitrosation by nitrite in the mouse heart during ischemia. J Biol Chem. 2017;292(35):14486-14495.

28. Kashiwagi S, et al. eNOS phosphorylation on serine 1176 affects insulin sensitivity and adiposity. Biochem Biophys Res Commun. 2013;431(2):284-290

29. Riachi M, Himms-Hagen J, Harper ME. Percent relative cumulative frequency analysis in indirect calorimetry: application to studies of transgenic mice. Can J Physiol Pharmacol. 2004;82(12):1075-1083.

30. Seth D, Stamler JS. The SNO-proteome: causation and classifications. Curr Opin Chem Biol. 2011;15(1):129-136.

31. Smith BC, Marletta MA. Mechanisms of S-nitrosothiol formation and selectivity in nitric oxide signaling. Curr Opin Chem Biol. 2012;16(5-6):498-506.

32. Gould N, Doulias PT, Tenopoulou M, Raju K, Ischiropoulos H. Regulation of protein function and signaling by reversible cysteine S-nitrosylation. J Biol Chem. 2013;288(37):26473-26479.

33. Fu S, et al. Aberrant lipid metabolism disrupts calcium homeostasis causing liver endoplasmic reticulum stress in obesity. Nature. 2011;473(7348):528-531. 
34. Muoio DM, Newgard CB. Mechanisms of disease: molecular and metabolic mechanisms of insulin resistance and beta-cell failure in type 2 diabetes. Nat Rev Mol Cell Biol. 2008;9(3):193-205.

35. Postic C, Girard J. Contribution of de novo fatty acid synthesis to hepatic steatosis and insulin resistance: lessons from genetically engineered mice. J Clin Invest. 2008;118(3):829-838.

36. Nozaki Y, et al. Deficiency of eNOS exacerbates early-stage NAFLD pathogenesis by changing the fat distribution. BMC Gastroenterol. 2015;15:177.

37. Baron AD, Zhu JS, Marshall S, Irsula O, Brechtel G, Keech C. Insulin resistance after hypertension induced by the nitric oxide synthesis inhibitor L-NMMA in rats. Am J Physiol. 1995;269(4 Pt 1):E709-E715.

38. Sheldon RD, Padilla J, Jenkins NT, Laughlin MH, Rector RS. Chronic NOS inhibition accelerates NAFLD progression in an obese rat model. Am J Physiol Gastrointest Liver Physiol. 2015;308(6):G540-G549.

39. Guan HP, Goldstein JL, Brown MS, Liang G. Accelerated fatty acid oxidation in muscle averts fasting-induced hepatic steatosis in SJL/J mice. J Biol Chem. 2009;284(36):24644-24652.

40. Frey AJ, Feldman DR, Trefely S, Worth AJ, Basu SS, Snyder NW. LC-quadrupole/Orbitrap high-resolution mass spectrometry enables stable isotope-resolved simultaneous quantification and ${ }^{13} \mathrm{C}$-isotopic labeling of acyl-coenzyme A thioesters. Anal Bioanal Chem. 2016;408(13):3651-3658.

41. Snyder NW, et al. Production of stable isotope-labeled acyl-coenzyme A thioesters by yeast stable isotope labeling by essential nutrients in cell culture. Anal Biochem. 2015;474:59-65.

42. Pinheiro LC, Ferreira GC, Vilalva KH, Toledo JC, Tanus-Santos JE. Contrasting effects of low versus high ascorbate doses on blood pressure responses to oral nitrite in L-NAME-induced hypertension. Nitric Oxide. 2018;74:65-73. 